\title{
Numerical investigation of conjugate natural convection heat transfer in a square porous cavity heated partially from left sidewall
}

\author{
Khaled Al-Farhany ${ }^{1 *}$, Ammar Abdulkadhim ${ }^{2}$ \\ ${ }^{1}$ Department of Mechanical Engineering, University of Al-Qadisiyah, Al-Qadisiyah 58001, Iraq \\ ${ }^{2}$ Air conditioning and Refrigeration Techniques Engineering Department, Al-Mustaqbal University College, Babylon 58001, \\ Iraq
}

Corresponding Author Email: khaled.alfarhany@qu.edu.iq

https://doi.org/10.18280/ijht.360132

Received: 6 December 2017

Accepted: 5 March 2018

\section{Keywords:}

natural convection, conjugate, porous, enclosure, COMSOL.

\begin{abstract}
The conjugate natural convection heat transfer in a partially heated square porous enclosure had been studied numerically. The governing dimensionless equations are solved using COMSOL Multiphysics and Darcy model assumed to be used. The considering dimensionless parameters are modified Rayleigh number, finite wall thickness, thermal conductivity ratio and the heat source length. The results are presented in terms of streamlines, isotherms and local and average Nusselt number. The results indicate that; the heat transfer can be enhanced by increasing the modified Rayleigh number. When the heat source length increases, the local Nusselt number of fluid phase increases, while, a reverse behavior of the local Nusselt number along the heat source is found. As the Rayleigh number increase, the local Nusselt number for both fluid and solid phase increases, therefore, the heat transfer rate will be enhanced. On the other hand, when the thermal conductivity ratio increase, the local Nusselt number for the fluid phase increases, and the local Nusselt number along the heated wall decreases.
\end{abstract}

\section{INTRODUCTION}

A large body of research has appeared with respect to investigations of convective heat transfer in vertical surfaces and rectangular porous cavities with surface temperatures. The classical Darcy formulation and Non-Darcy formulations have been used.

Recently, many investigations undertaken on natural convection in enclosures filled with porous media [1-7]. On the other hand, the conjugate heat convection in enclosures filled with porous medium has evoked much interest by many researchers due to its various practical applications such as cold storage installations and high performance insulation for buildings. These areas have been thoroughly reviewed recently by Nield and Bejan [8]. Das et al. [9] reviewed the studies regarding the natural convection heat transfer in porous enclosure for different shapes. Mbaye et al. [10] had investigated analytical and numerical studies of natural convection in an inclined porous layer boarded by a finitethickness wall. The horizontal walls were insulated, while the vertical sidewalls kept at constant heat flux $(\overline{\bar{q}})$. Chang and Lin [11] examined the heat transfer effects on conjugate natural convection in a porous enclosure surrounded by finite walls. The results showed that the overall heat transfer rate from the hot side to cold side of the enclosure decreased due to the heat conduction on the wall. Baytas et al. [12] studied numerically, the effects of conjugate heat convection in a square porous cavity bounded by two horizontal conductive finite thickness walls, and two vertical walls at different uniform temperatures. They concluded that, for large values of the conductivity ratio, the flow characteristics were significantly influenced due to the strong coupling effects between fluid-saturated porous medium and the solid walls. Al-Farhany and Turan [13] performed a numerical analysis of natural convective heat transfer in an inclined porous rectangular using Finite Volume Method for various inclination angles $(0 \leq \varphi \leq 85)$ and aspect ratio $(2 \leq \mathrm{A} \leq 5)$. The results show that, both of the average Sherwood and Nusselt numbers decrease, when the angle of inclination increases, moreover they decrease when the aspect ratio increases. Saeid [14] studied numerically the effects of conjugate natural convective heat transfer in a twodimensional vertical porous enclosure. The horizontal walls were kept insulated, while the outer surfaces of the vertical walls with a finite thickness were isothermal at different temperatures. The numerical work covered a wide range of governing parameters. It was found that the average Nusselt numbers increased when the Rayleigh number and the thermal conductivity increased, while the Nusselt numbers decreased when the wall thickness increased. Al-Amiri et al. [15] studied numerically, the conjugate natural convective heat transfer in a fluid-saturated porous enclosure. The porosity of the porous medium $(\varepsilon)$ were investigated as well as the effect of Darcy number. Saleh and Hashim [16] studied the effects of internal heat generation on the natural convection heat transfer in porous cavity. Rayleigh number, thermal conductivity ratio and the wall thickness were used as governing parameters. Their conclusions were, the maximum fluid temperature increase when, the thickness of a solid wall decrease. Furthermore, it increase when the thermal conductivity ratio increase.

Bhuvaneswari et al. [17], investigated numerically the effect of aspect ratio in a porous enclosure with partially active walls. The generalized model have been used and the governing equations solved by using Finite volume method with SIMPLE algorithm. Their results shows that, the location of heating and cooling area influence significantly in the rate 
of heat transfer and the flow patterns in the enclosure. Numerical investigation of conjugate natural heat convection in a differentially heated square enclosure done by Roslan et al [18]. A conductive polygon object used for various Rayleigh number. The results display that polygon object impact on the heat transfer rate. Hossain and Wilson solved using finite difference approach the natural convective fluid flow in a porous rectangular enclosure. All the walls assumed to be Non-isothermal walls with effects of the internal heat generation. The authors studied the effect of porosity, internal heat generation and the rate of heat transfer. Saleh et al. [19] studied the effect of conduction wall from the bottom of a porous square cavity on the rate of heat transfer. COMSOL Multiphysics software is used to solve the non-dimensional governing equations. The results indicate that, when the Rayleigh number and/or thermal conductivity ratio increasing, in addition to decreasing the bottom wall thickness, the circulations of fluid flow and the rate of heat transfer rate increasing. An in-house code based on finite volume method with SIMPLE/SIMPLER algorithm made by Al-Farhany and Turan [20] to study the heat convection in a square porous enclosure with finite wall thickness from two sides. A good correlation equation presented to predict average Nusselt number on the interface of left wall in terms of the governing parameters. More works that are complicated done by AlFarhany and A. Turan [21] to investigate the effects of variable porosity. Revnic et al. [22] used finite difference method to solve numerically free convection flow in a bidisperes porous medium in a square cavity. Natural heat transfer in an inclined porous square enclosure with finite walls thickness from both sides have been investigated numerically by Ahmed et al. [23]. The left sidewall heated partially when the other walls insulated. The results indicate that, when the inclination angle $(\varphi)$ increases (from $0^{\circ}$ to $45^{\circ}$ ), the average Nusselt number increases. Also, when $\varphi=60^{\circ}$, the average Nusselt number decreases slightly while, it decreases significantly when $\varphi=$ $90^{\circ}$.

Ma et al. [24] studied numerically, two-dimensional natural convection in a porous square cavity by using Lattice Boltzmann method. The cavity was heated from bottom and the other walls kept at cold temperature. The two vertical cold walls assumed to be varied in its length size. The effect of Darcy number, Rayleigh number, the length of the dimensionless, and porosity on the natural convective heat transfer rate had been included in the study. The results shows that the Darcy and Rayleigh numbers have significant effect on heat transfer as it enhanced as they increase.

It can be noticed from the literature review and according to the best of the authors' knowledge; there are limitations in the studies regarding partially active walls of conductionconvection porous enclosures. The main objective of the present work is to study the conjugate natural convection heat transfer in a porous enclosure partially heated from its left side wall.

\section{MATHEMATICAL FORMULATION}

In this paper, two-dimensional congregate of natural convicted heat transfer in porous cavity have been studied numerically with the effect of the partially heated conduction on vertical wall as shown in Figure 1. The horizontal walls are adiabatic while, different cases have been investigations with different partially heated walls on the left side while, the right walls are kept at constant cold temperature.

The Darcy model was chosen to solve the governing equations. Saturated water used as a working fluid and the porous medium is assumed to be homogeneous and isotropic. y

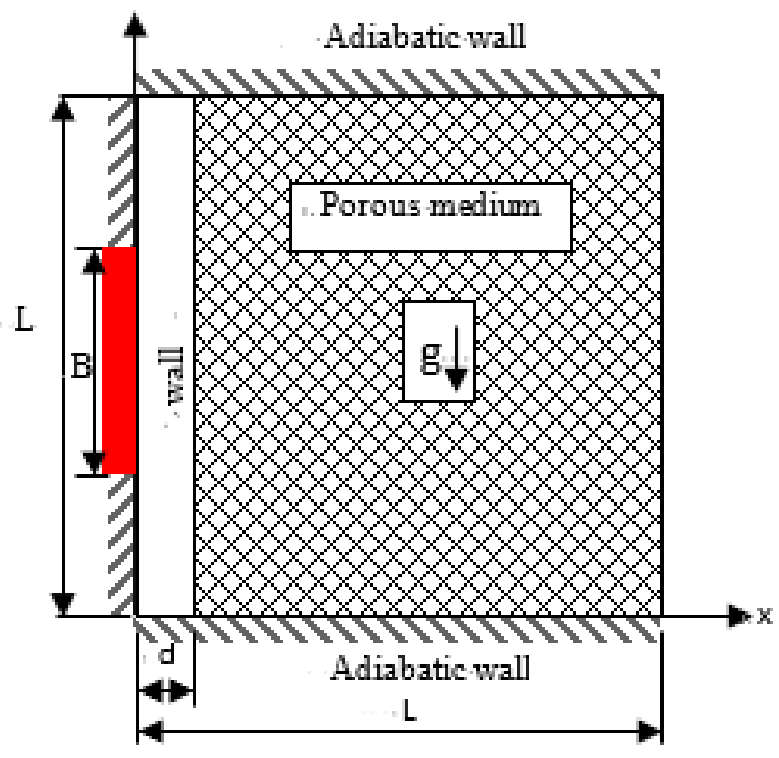

Figure 1. Schematic diagram of the partially heated porous enclosure

All the properties have been assumed to be constant except the density. The flow is driven by buoyancy effect due to temperature variations only. The density variations are described by the Boussinesq approximation:

$\rho=\rho\left[1-\beta_{T}\left(T-T_{o}\right)\right]$

where $\beta_{T}=-\frac{1}{\rho}\left(\frac{\partial \rho}{\partial T}\right)_{P}$ is the thermal expansion coefficient.

The Continuity, momentum and energy of two-dimensional steady state natural convection in porous cavity equations are:

Continuity equation:

$\frac{\partial U}{\partial X}+\frac{\partial V}{\partial Y}=0$

X-momentum equation:

$\frac{\partial U}{\partial X}+\frac{\partial U}{\partial Y}=0$

Y-momentum equation:

$\frac{\partial U}{\partial Y}+\frac{\partial U}{\partial X}=-\frac{g \beta \cdot \mathrm{K}}{v} \frac{\partial P}{\partial X}$

Energy equation:

$U \cdot \frac{\partial T}{\partial X}+V \cdot \frac{\partial T}{\partial Y}=\frac{\partial^{2} T}{\partial X^{2}}+\frac{\partial^{2} T}{\partial Y^{2}}$ 
and the energy equation at the walls:

$$
\frac{\partial^{2} T_{W}}{\partial X^{2}}+\frac{\partial^{2} T_{W}}{\partial Y^{2}}=0
$$

The heat transfer at the walls are defined as in the following:

$$
\left.N u=\int_{0}^{1}-\frac{\partial T}{\partial X}\right) \cdot \partial Y
$$

where $\mathrm{Nu}$ is the average Nusselt number.

The non-dimensional parameters are:

$$
\begin{aligned}
& X=\frac{x}{L}, Y=\frac{y}{L}, D=\frac{d}{L}, U=\frac{u L}{\alpha}, \\
& V=\frac{v L}{\alpha}, P=\frac{p L^{2}}{\rho \alpha^{2}}, T=\frac{\bar{T}-\overline{T_{c}}}{\overline{T_{h}}-\overline{T_{c}}} \\
& R a=\frac{g \beta_{T} \Delta T K L}{v \alpha}, k_{r}=\frac{k_{w}}{k_{f}} \\
& k_{\text {eff }}=\varepsilon k_{f}+(1-\varepsilon) k_{s}
\end{aligned}
$$

Equations (2)-(7) are solved using non-dimensional initial boundary conditions:

$$
\begin{array}{lll}
\text { at } X=D ; & U=V=0 ; & \frac{\partial T_{w}}{\partial X}=k_{r} \frac{\partial T}{\partial X} \\
\text { at } X=1 ; & U=V=0 ; & T_{c}=0 \\
\text { at } X=0 ; & T_{h}=1 & \\
\text { at } Y=0,1 ; & U=V=0 ; & \frac{\partial T}{\partial Y}=0
\end{array}
$$

CFD software package COMSOL Multiphysics used to solved the governing equations along with the boundary conditions. COMSOL Multiphysics is a finite element analysis, solver, and simulation software package for various physics and engineering applications. In this study, triangular mesh generation on square enclosure filled with saturated porous media containing finite wall thickness from one wall. The triangular mesh distribution is shown in Figure 2 for determination whether the scheme of mesh is sufficient and to ensure that the results are grid independent. Grid sensitivity tests were presented such as normal, fine, finer, extra fine, and extremely as tabulated in Table 1 . Considering the accuracy and CPU time, an extra finer mesh size was selected for all the computations done in the present work.

Table 1. Grid sensitivity check, for $\mathrm{Ra}=10^{3}, \mathrm{D}=0.1, \mathrm{~K}_{\mathrm{r}}=1$

\begin{tabular}{ccc}
\hline \hline Predefined Mesh Size & $\overline{N u_{f}}$ & CPU time sec \\
\hline Extremely fine & 1.9679 & 272 \\
Extra fine & 1.9384 & 62 \\
Finer & 1.8781 & 15 \\
Fine & 1.8418 & 10 \\
Normal & 1.8380 & 7 \\
\hline \hline
\end{tabular}

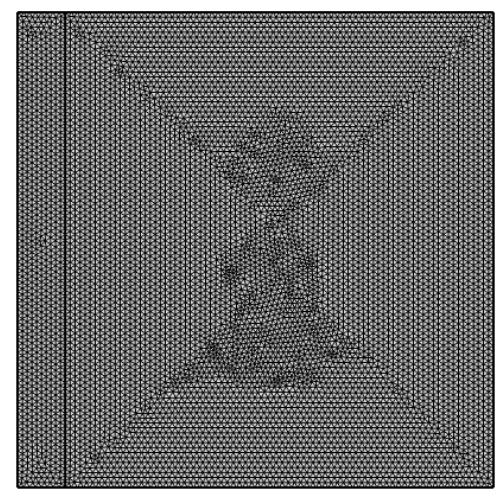

Figure 2. Mesh Distribution of the partially heated porous enclosure

\section{CODE VALIDATION}

The present numerical results obtained from simulation using COMSOL 5.2a are validated with significant researchers to ensure the accuracy of the present program. The streamlines and isotherms contours of the present work are validated with those of Saeid work [14] for $\left[\mathrm{Ra}=1000, \mathrm{D}=0.02\right.$, and $\mathrm{K}_{\mathrm{r}}=$ 1]. Comparisons give a good agreement between the previous and present results as shown in Figure 3. Furthermore, The average nusselt number of the present work also have been validated with Beckermann et al. [25], Moya et al. [26], Nithiarasu et al. [1], Al-Farhany, Turan [21] and Ahmed et al [23] as shown in Table 2.

Table 2. Comparison of average Nusselt number with some previous results for Darcy model

\begin{tabular}{ccc}
\hline \hline Author & $\mathrm{Ra}=10$ & $\mathrm{Ra}=100$ \\
\hline Nithiarasu et al. [1] & 1.08 & 3.02 \\
Al-Farhany, Turan [21] & 1.119 & 3.05 \\
Ahmed et al. [23] & 1.093 & 3.01 \\
Beckermann et al. [25] & & 3.113 \\
Moya et al. [26] & 1.065 & 2.801 \\
Present Work & $\mathbf{1 . 0 8}$ & $\mathbf{3 . 0 2}$ \\
\hline \hline
\end{tabular}
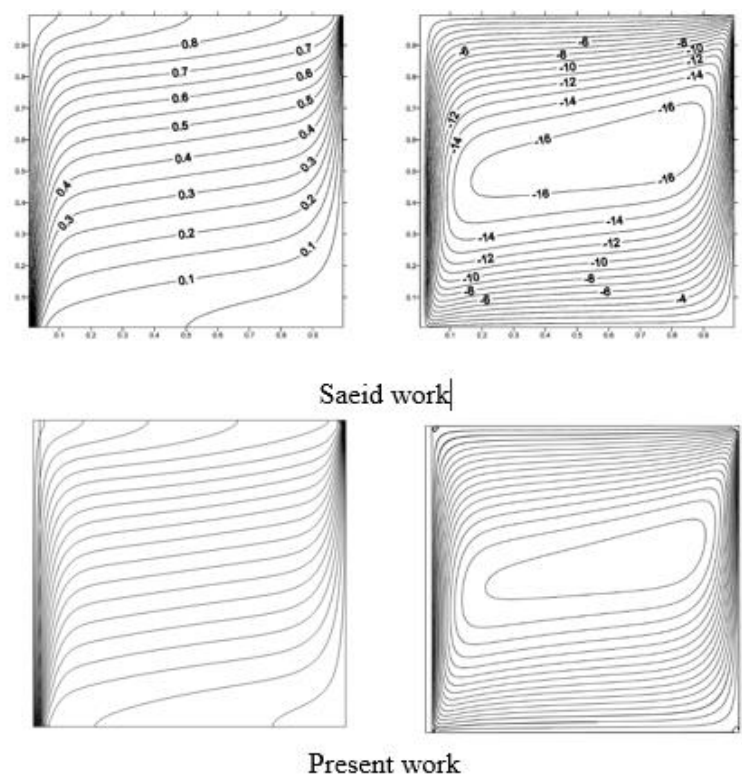

Figure 3. Comparison of streamlines and isotherms between Saeid [24] work (top) and the present work (bottom) when $\mathrm{Ra}=10^{3}, \mathrm{D}=0.02$, and $\mathrm{K}_{\mathrm{r}}=1$ 


\section{RESULTS}

\subsection{Modified rayleigh number}
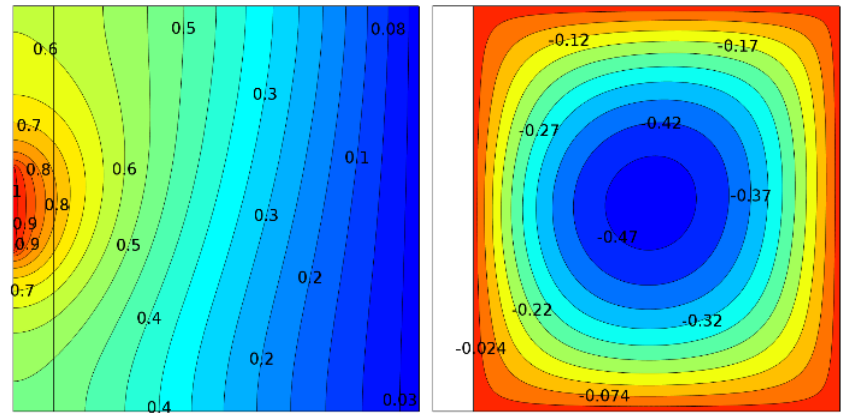

a. $\quad \mathrm{Ra}=10, \overline{N u_{f}}=0.75577, \overline{N u_{w}}=3.0109$
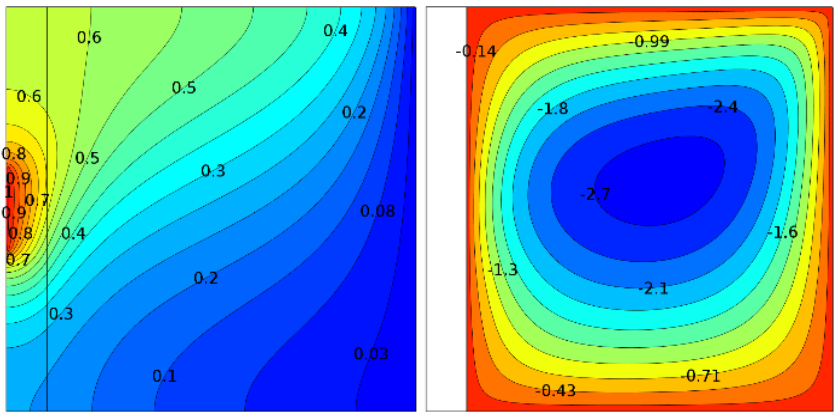

b. $\quad \mathrm{Ra}=100, \overline{N u_{f}}=1.2024, \overline{N u_{w}}=4.8974$
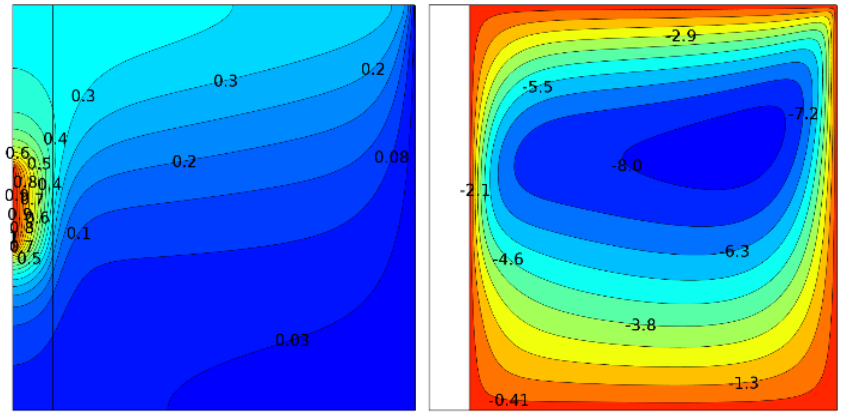

c. $\quad \mathrm{Ra}=1000, \overline{N u_{f}}=1.9384, \overline{N u_{w}}=8.2210$

Figure 4. Isotherm (left) and Streamlines (right) for various modified Rayleigh number, $\mathrm{D}=0.1, \mathrm{~K}_{\mathrm{r}}=1$, and $\mathrm{B}=0.2$

Figure 4 demonstrates the isotherms and streamlines for various modified Rayleigh number for $\mathrm{D}=0.1, \mathrm{~K}_{\mathrm{r}}=1$, and $\mathrm{B}=0.2$. It is observed that, when modified Rayleigh number increases, the vortex strength increases for all cases. The effects on the streamline behaviors is very pronounced at high Rayleigh number. These influences on the vortex strength are proportional to buoyancy force increases due to increasing in heat transfer rates. It can be noted that when modified Rayleigh number increase, the Nusselt number increase. For example, when modified Rayleigh number $(\mathrm{Ra}=10)$, the Nusselt numbers are $\overline{N u_{f}}=0.75577, \overline{N u_{w}}=3.0109$ while the Nusselt numbers are $\overline{N u_{f}}=1.9384, \overline{N u_{w}}=8.2210$ when $\mathrm{Ra}=1000$. The physical reason behind this phenomenon is that; when the modified Rayleigh number increases, the flow circulation intensity increases. With respect to isotherms, it can be seen that when modified Rayleigh number (at $\mathrm{Ra}=$ 10), the isotherms have a uniform shape. This is due to weak effect of convective flow and in this case, the conductive heat transfer is dominant. However, when modified Rayleigh number increases (at $\mathrm{Ra}=1000$ ), the isotherms change their shapes obviously due to strong effect of convection heat transfer.

\subsection{Dimensionless wall thickness effects}

Figure 5 illustrates the isotherms and streamlines for various dimensionless wall thickness $\mathrm{Ra}=1000, \mathrm{~K}_{\mathrm{r}}=1$, and, $\mathrm{B}=0.2$ ). The flow circulation strength in the porous medium is higher with thin walls while it was lower with thick wall as shown in Figure 5. It may be noted that as the dimensionless wall thickness increases, the Nusselt number decrease, and that happen because the conduction heat transfer is dominant with increasing wall thickness. For the isotherms contours; it may be noted that; the isotherms pattern becomes more uniform as the walls thickness increases. That happened because the effect of the conductive heat transfer mode becomes more dominate. Moreover, the isotherms pattern demonstrates that the heat transfer is taken place from the hot left sidewall at the middle towards the cold right sidewall because of the large temperature gradient and this result matches with the problem boundary conditions.
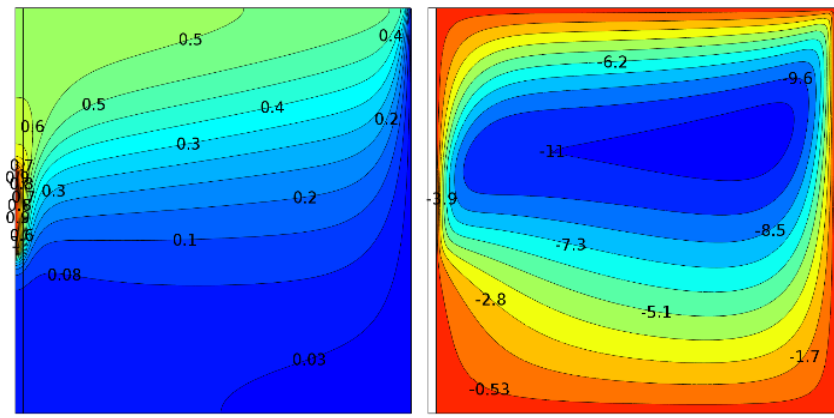

a. $\quad \mathrm{D}=0.02, \overline{N u_{f}}=4.1316, \overline{N u_{w}}=18.943$
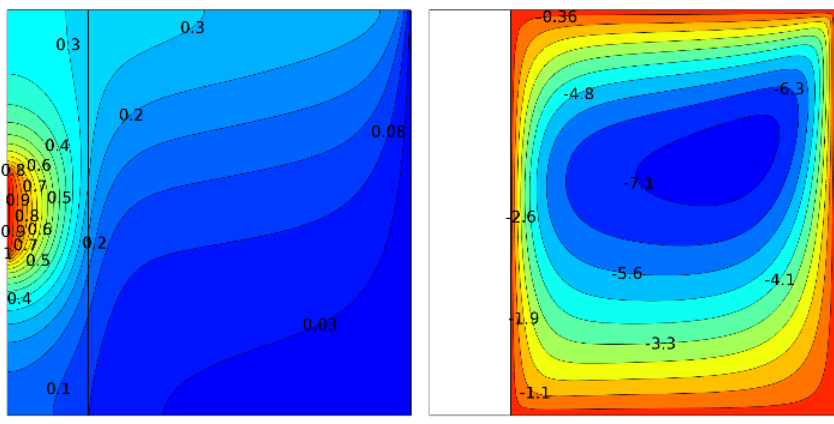

b. $\quad \mathrm{D}=0.2, \overline{N u_{f}}=1.4226, \overline{N u_{w}}=8.8703$
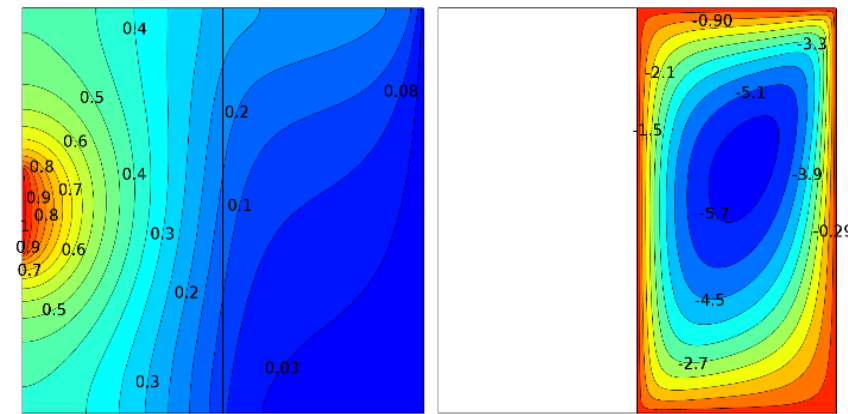

$$
\text { c. } \quad \mathrm{D}=0.5, \overline{N u_{f}}=0.95096, \overline{N u_{w}}=3.8650
$$

Figure 5. Isotherm (left) and Streamlines (right) for various dimensionless wall thickness, $\mathrm{Ra}=1000, \mathrm{~K}_{\mathrm{r}}=1$, and $\mathrm{B}=0.2$ 


\subsection{Thermal Conductivity Ratio effects}

Figure 6 shows the effect of thermal conductivity ratio on heat transfer rate at $\mathrm{Ra}=1000, \mathrm{D}=0.1$ and $\mathrm{B}=0.2$. In this paper, the thermal conductivity ratio $\left(K_{\mathrm{r}}\right)$ is defined as the ratio of the thermal conductivity of solid walls to the thermal conductivity of the fluid. It can be obtained that; when the thermal conductivity ratio is small, the thermal resistance in the left sidewall is high. Therefore, the average Nusselt number at solid walls is high, while the average Nusselt number of fluids is low. On the contrary of that can be seen when the thermal conductivity ratio increases the thermal conductivity of solid walls increases. For that reason, the average Nusselt number at solid walls decreases. On the other hand, the average Nusselt number on the fluid side increases with the increasing of thermal conductivity ratio. This result demonstrates that; the heat transfer mechanism inside the enclosure is converted from conduction when $\mathrm{K}_{\mathrm{r}}$ is low to convection when $\mathrm{K}_{\mathrm{r}}$ is high.
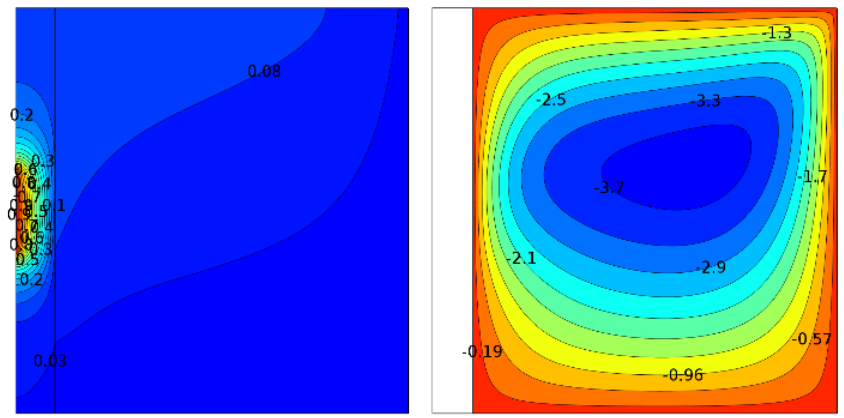

a. $\quad \mathrm{K}_{\mathrm{r}}=0.1, \overline{N u_{f}}=1.4657, \overline{N u_{w}}=10.961$
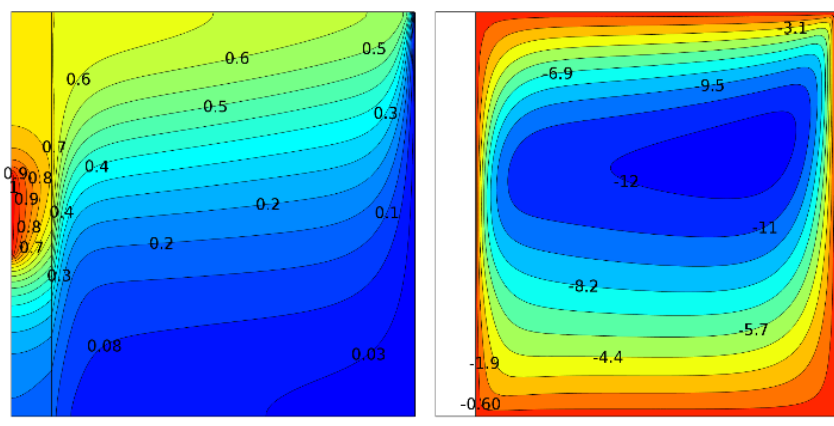

b. $\mathrm{K}_{\mathrm{r}}=5, \overline{N u_{f}}=3.1341, \overline{N u_{w}}=4.5560$
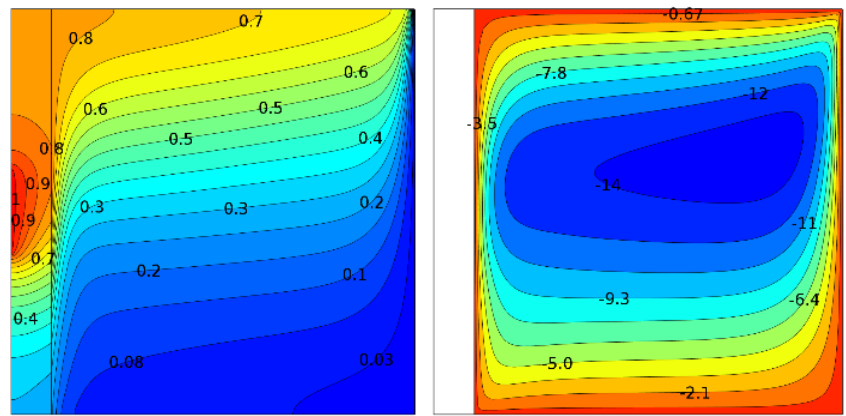

c. $\quad \mathrm{K}_{\mathrm{r}}=10, \overline{N u_{f}}=3.8093, \overline{N u_{w}}=3.0052$

Figure 6. Isotherm (left) and Streamlines (right) for various thermal conductivity ratio, $\mathrm{Ra}=1000, \mathrm{D}=0.1$, and $\mathrm{B}=0.2$

\subsection{Heat source length effects}

Figure 7 shows streamlines and isotherms for various values of dimensionless heat source length (B) at $\mathrm{Ra}=1000, \mathrm{D}=0.1$, and $\mathrm{K}_{\mathrm{r}}=1$. It can be observed that the average Nusselt number at solid walls decreases from $\overline{N u_{w}}=8.2210$ at $\mathrm{B}=0.2$ to $\overline{N u_{w}}=5.4808$ at $\mathrm{B}=0.8$. On the other hand, the average Nusselt number of fluids increases from $\overline{N u_{f}}=1.9384$ at B $=$ 0.2 to $\overline{N u_{f}}=4.4704$ at $\mathrm{B}=0.8$. The results indicate that, the convection of the porous medium enhances when the heat source length increases, while the average Nusselt number of solid walls decreases as the heat source length increases. Also, the isotherms distribution is strongly dependent on the heat source length. It can be notice that the heat source on the hot left sidewall transfers most of heat into the right cold wall due to the large gradient of temperature, as can be seen from the isotherms accumulated close to the right cold wall.
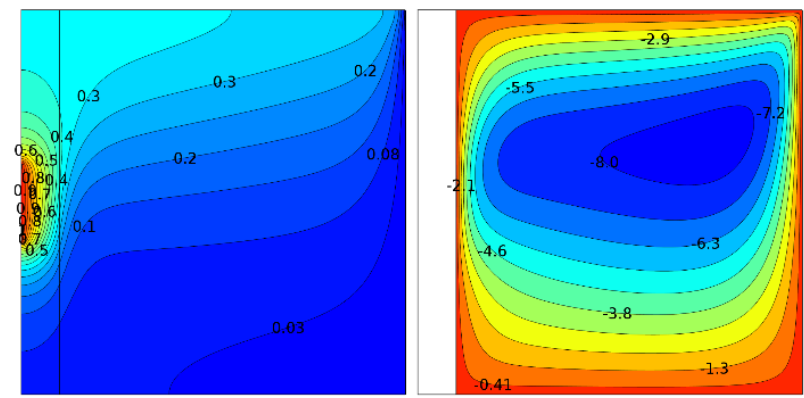

a. $\quad \mathrm{B}=0.2, \overline{N u_{f}}=1.9384, \overline{N u_{w}}=8.2210$
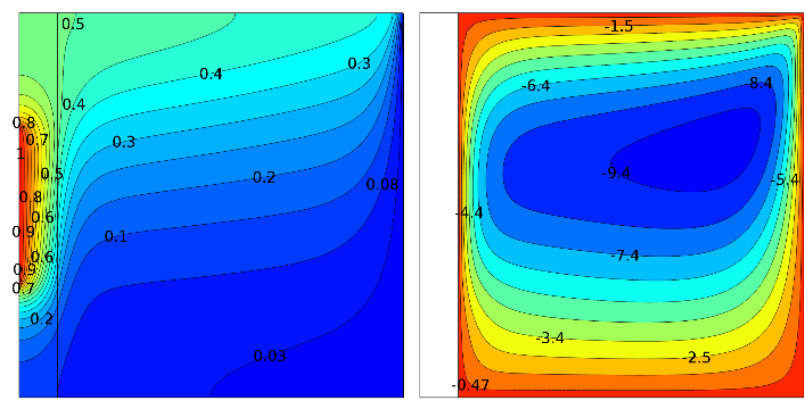

b. $\quad \mathrm{B}=0.4, \overline{N u_{f}}=2.9302, \overline{N u_{w}}=6.7703$
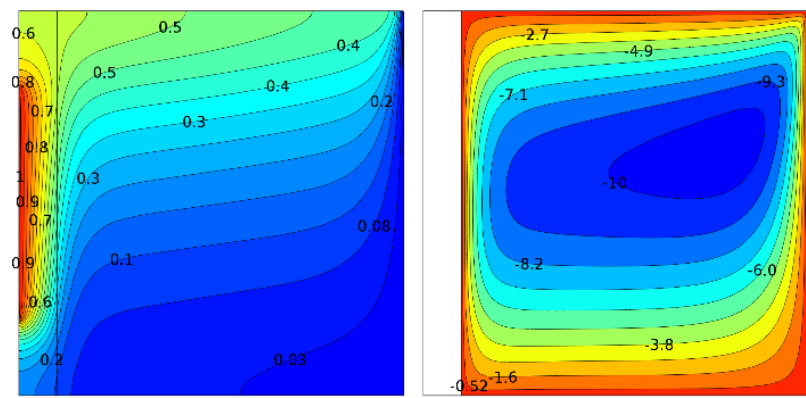

c. $\quad \mathrm{B}=0.6, \overline{N u_{f}}=3.7803, \overline{N u_{w}}=6.0427$
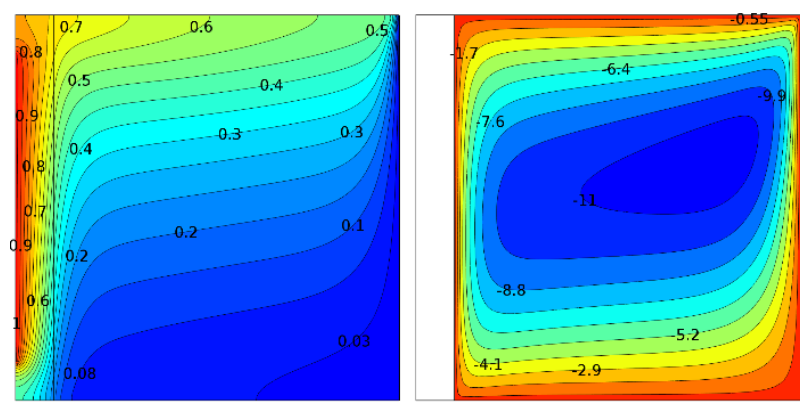

d. $\quad \mathrm{B}=0.8, \overline{N u_{f}}=4.4704, \overline{N u_{w}}=5.4808$

Figure 7. Isotherm (left) and Streamlines (right) for various heat source length, $\mathrm{Ra}=1000, \mathrm{D}=0.1$, and $\mathrm{K}_{\mathrm{r}}=1$ 


\subsection{Local Nusselt Number results}

The local Nusselt number profile of the fluid and the solid phase presented in Figure 8 for various values of modified Rayleigh number at $\mathrm{D}=0.1, \mathrm{~K}_{\mathrm{r}}=1$, and $\mathrm{B}=0.2$. As expected, the heat transfer rate enhanced significantly, when $\mathrm{Ra}$

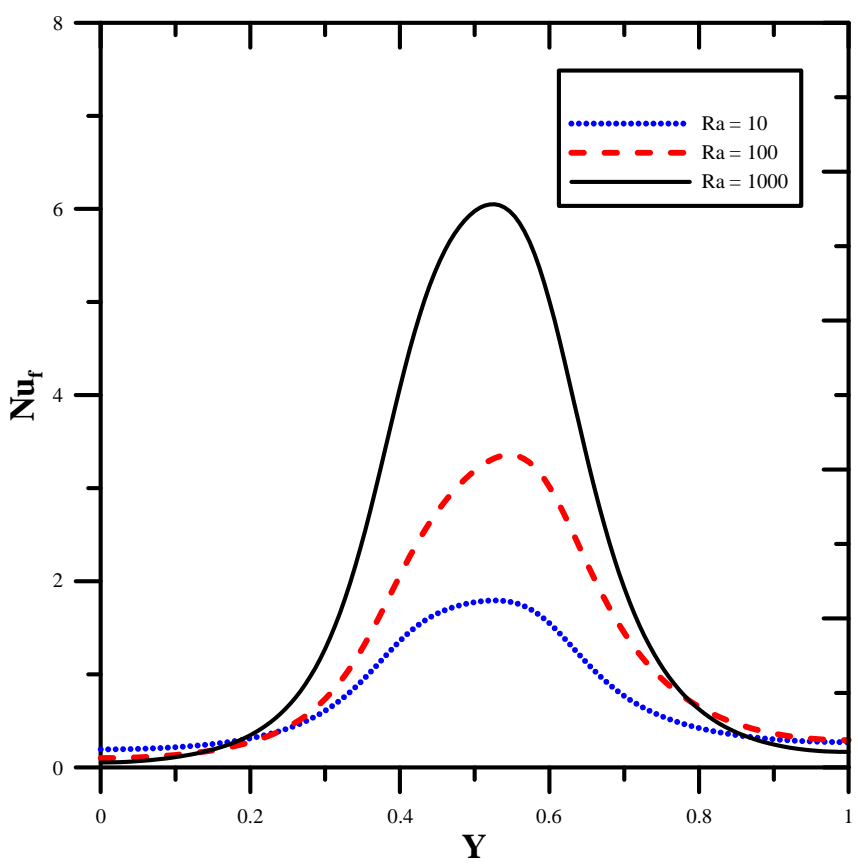

increases from 100 to 1000 due to the increasing of buoyancy and natural convection flow within the enclosure. Same behaviors can be seen when the thermal conductivity ratio increases as well as the increasing of the length of hot wall as shown in Figures 9 and 10 respectively.

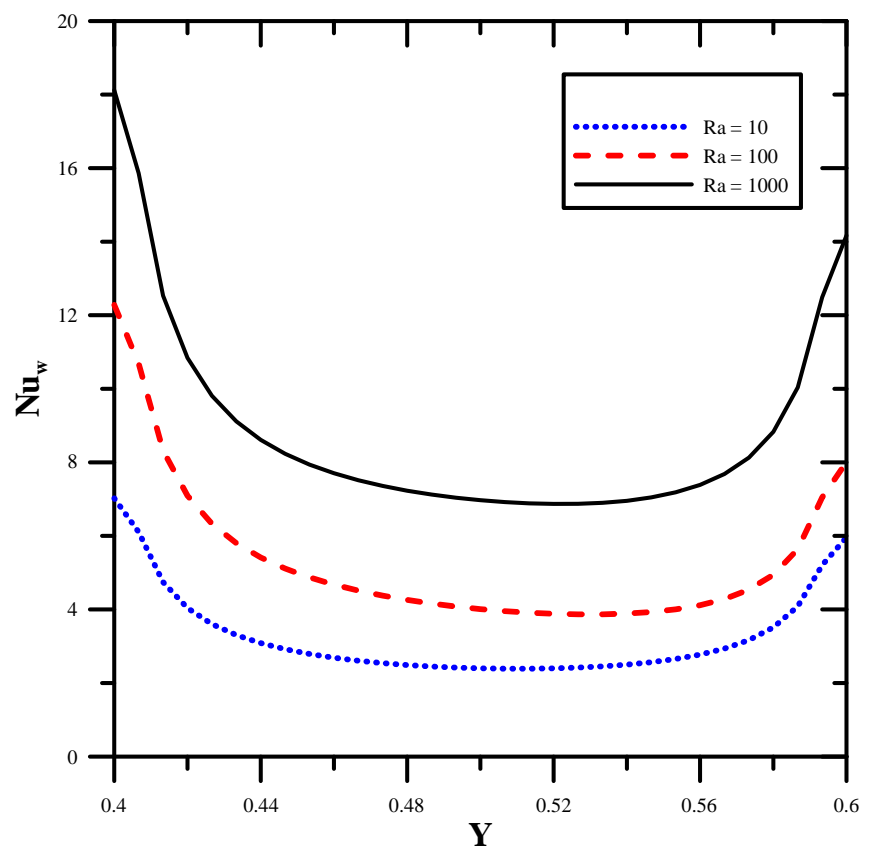

Figure 8. Profile of local Nusselt number for fluid phase (top) and along the thermally active heated wall (bottom) for various modified Rayleigh number at $\mathrm{D}=0.1, \mathrm{~K}_{\mathrm{r}}=1$ and $\mathrm{B}=0.2$
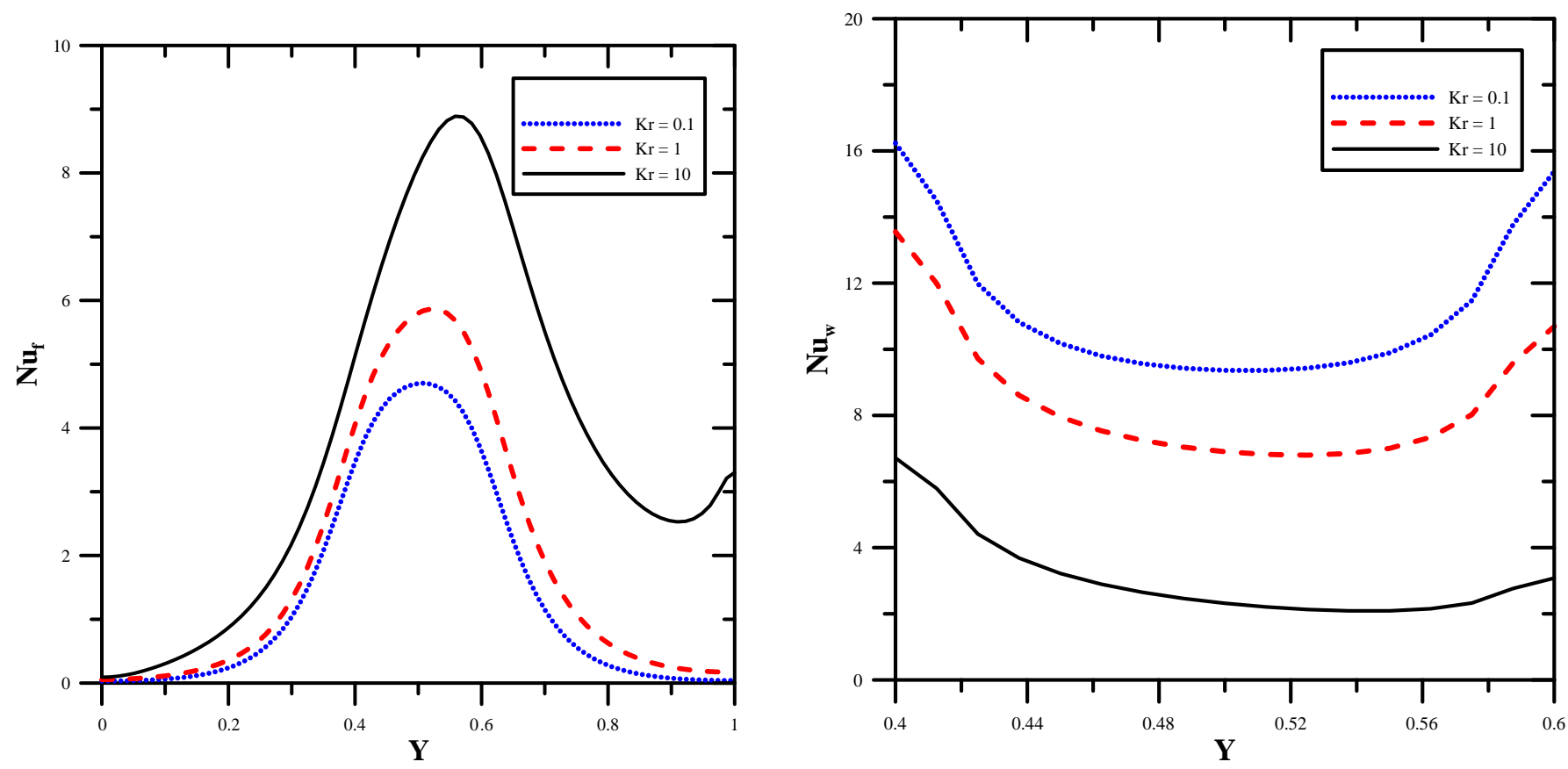

Figure 9. Profile of local Nusselt number for fluid phase and along the thermally active heated wall for various thermal conductivity ratio, $\mathrm{D}=0.1, \mathrm{Ra}=1000$, and $\mathrm{B}=0.2$. 

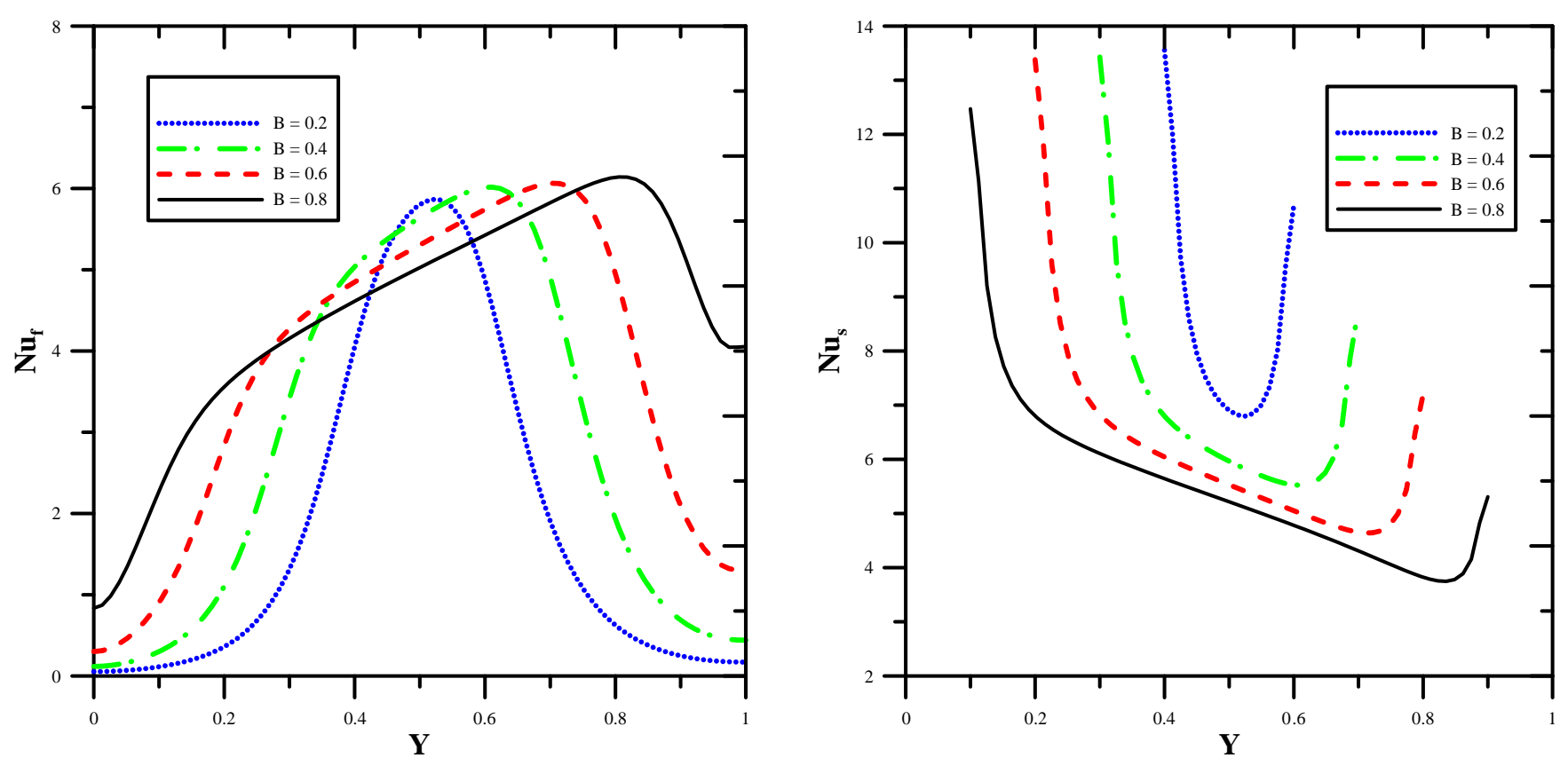

Figure 10. Profile of local Nusselt number for fluid phase (top) and along the thermally active heated wall (bottom) for various hot wall length, at $\mathrm{K}_{\mathrm{r}}=1, \mathrm{Ra}=1000$, and $\mathrm{D}=0.1$

\section{CONCLUSIONS}

Numerical study of natural convective heat transfer in a porous enclosure partially heated from its left sidewall using COMSOL Multiphasics 5.2a software. The results are presented in wide range of the dimensionless numbers including, modified Rayleigh number $(10 \leq R a \leq 1000)$, dimensionless wall thickness $(0.02 \leq D \leq 0.5)$, thermal conductivity ratio $(0.1 \leq K r \leq 10)$, and heat source length $(0.2 \leq B \leq 0.8)$.

The main results shows that when the wall thickness increases, the convection mechanism will be converted conduction into mode. For that reason, the Nusselt number reduce. Furthermore, as the thermal conductivity ratio increases, the local Nusselt number for the fluid phase increases, while a reverse behavior shown for local Nusselt number along the heated wall. Moreover, when the Rayleigh number increases, the local Nusselt number for both fluid and solid phase increases. Finally, when the heat source length increases, the local Nusselt number of fluid phase increases while, a reverse behavior of the local Nusselt number profiles along the heat source.

\section{REFERENCES}

[1] Nithiarasu P, Seetharamu K, Sundararajan T. (1997). Non-Darcy double-diffusive natural convection in axisymmetric fluid saturated porous cavities. Heat and mass transfer 32(6): 427-433.

[2] Basak T, et al. (2006). Natural convection in a square cavity filled with a porous medium: effects of various thermal boundary conditions. International Journal of Heat and Mass Transfer 49(7): 1430-1441.

[3] Oztop HF. (2007). Natural convection in partially cooled and inclined porous rectangular enclosures. International Journal of Thermal Sciences 46(2): 149-156.

[4] Sathiyamoorthy M, et al. (2007). Steady natural convection flow in a square cavity filled with a porous medium for linearly heated side wall (s). International Journal of Heat and Mass Transfer 50(9): 1892-1901.

[5] Bhattacharya M, Basak T. (2013). On multiple steady states for natural convection (low Prandtl number fluid) within porous square enclosures: Effect of nonuniformity of wall temperatures. International Journal of Heat and Mass Transfer 59: 230-246.

[6] Singh AK, et al. (2015). Role of entropy generation on thermal management during natural convection in tilted porous square cavities. Journal of the Taiwan Institute of Chemical Engineers 50: 153-172.

[7] Siavashi M, Bordbar V, Rahnama P. (2017). Heat transfer and entropy generation study of non-Darcy double-diffusive natural convection in inclined porous enclosures with different source configurations. Applied Thermal Engineering 110: 1462-1475.

[8] Nield DA, Bejan A, Nield-Bejan (2006). Convection in porous media. 3, Springer.

[9] Das D, Roy M, Basak T. (2017). Studies on natural convection within enclosures of various (non-square) shapes-A review. International Journal of Heat and Mass Transfer 106: 356-406.

[10] Mbaye M, Bilgen E, Vasseur P. (1993). Naturalconvection heat transfer in an inclined porous layer boarded by a finite-thickness wall. International journal of heat and fluid flow 14(3): 284-291.

[11] Chang WJ, Lin H.C. (1994). Natural convection in a finite wall rectangular cavity filled with an anisotropic porous medium. International Journal of Heat and Mass Transfer 37(2): 303-312.

[12] Baytaş A, et al. (2001). Conjugate natural convection in a square porous cavity. Heat and Mass transfer 37(4): 467-473.

[13] Al-Farhany K, Turan A. (2012). Numerical study of double diffusive natural convective heat and mass transfer in an inclined rectangular cavity filled with porous medium. International Communications in Heat and Mass Transfer 39(2): 174-181.

[14] Saeid NH. (2007). Conjugate natural convection in a 
porous enclosure: effect of conduction in one of the vertical walls. International Journal of Thermal Sciences 46(6): 531-539.

[15] Al-Amiri A, Khanafer K, Pop I. (2008). Steady-state conjugate natural convection in a fluid-saturated porous cavity. International Journal of Heat and Mass Transfer 51(17-18): 4260-4275.

[16] Saleh H, Hashim I. (2012). Conjugate natural convection in a porous enclosure with non-uniform heat generation. Transport in porous media 1-16.

[17] Bhuvaneswari M, Sivasankaran S, Kim YJ. (2011). Effect of aspect ratio on convection in a porous enclosure with partially active thermal walls. Computers \& Mathematics with Applications 62(10): 3844-3856.

[18] Roslan R, Saleh H, Hashim I. (2014). Natural convection in a differentially heated square enclosure with a solid polygon. The Scientific World Journal 2014.

[19] Saleh H, et al. (2011). Effect of conduction in bottom wall on Darcy-Bénard convection in a porous enclosure. Transport in porous media 88(3): 357-368.

[20] Al-Farhany K, Turan A. (2011). Unsteady conjugate natural convective heat transfer in a saturated porous square domain generalized model. Numerical Heat Transfer, Part A: Applications 60(9): 746-765.

[21] Al-Farhany K, Turan A. (2011). Non-Darcy effects on conjugate double-diffusive natural convection in a variable porous layer sandwiched by finite thickness walls. International Journal of Heat and Mass Transfer 54(13): 2868-2879.

[22] Revnic C, et al. (2009). Free convection in a square cavity filled with a bidisperse porous medium. International Journal of Thermal Sciences 48(10): 1876 1883.

[23] Ahmed SE, et al. (2016). Conjugate natural convection in an inclined square porous enclosure with finite wall thickness and partially heated from its left sidewall. Heat Transfer Research 47(4).

[24] Ma Z, et al. (2015). Numerical study of natural convection heat transfer in porous media square cavity with multiple cold walls based on LBM. Int. J. Heat Tech 33: 69-76.

[25] Beckermann C, Viskanta R, Ramadhyani S. (1986). A numerical study of non-Darcian natural convection in a vertical enclosure filled with a porous medium. Numerical Heat Transfer 10(6): 557-570.

[26] Moya SL, Ramos E, Sen M. (1987). Numerical study of natural convection in a tilted rectangular porous material. International Journal of Heat and Mass Transfer 30(4): 741-756.

\section{NOMENCLATURE}

dimensional heat source length $\mathrm{m}$

\section{Greek symbols}

$\alpha \quad$ effective thermal diffusivity, $\mathrm{m}^{2} \mathrm{~s}^{-1}$

$\beta_{\mathrm{T}} \quad$ coefficient of thermal expansion, $\mathrm{K}^{-1}$

$\varepsilon \quad$ porosity of the porous media

$v \quad$ kinematic viscosity, $\mathrm{m}^{2} \mathrm{~s}^{-1}$

$\rho$ density, $\mathrm{kg} \mathrm{m}^{-3}$

$\sigma \quad$ ratio of specific heats

\section{Subscripts}

$\begin{array}{ll}\text { c } & \text { cold } \\ \text { eff } & \text { effective } \\ \text { f } & \text { fluid } \\ \text { h } & \text { hot } \\ \text { s } & \text { solid } \\ \text { w } & \text { wall }\end{array}$

